\title{
Avaliação de crianças atendidas em follow-up: perfil epidemiológico e motor
}

\section{Evaluation of children at follow-up: epidemiological and motor profile}

\author{
Mayara Cruz Vargasi'; Ayrles Silva Gonçalves Barbosa Mendonça²; Aléxia Gabriela da Silva \\ Vieiral; Ana Beatriz da Costa Lameira²; Nely Sampaio Guinther2; Tiótrefis Gomes Fernandes²; \\ Ana Paula Guimarães Dias Corrêa ${ }^{3}$; Marcos Giovanni Santos Carvalho3; Roberta Lins Gonçalves ${ }^{2}$ \\ 1 Programa de Residência em Fisioterapia em Terapia Intensiva Neonatal - Universidade Federal do Amazonas - UFAM. Manaus, AM \\ - Brasil. \\ 2 Departamento de Fisioterapia - Universidade Federal do Amazonas - UFAM. Manaus, AM - Brasil. \\ 3 Departamento de Fisioterapia, Maternidade Balbina Mestrinho. Manaus, AM - Brasil. \\ Endereço para Correspondência: \\ Mayara Cruz Vargas \\ Av. Jornalista Humberto Calderaro Filho, 2200. \\ Ed. Village Vert. Apt. 403. Adrianópolis. \\ 69057-015 - Manaus - AM [Brasil]. \\ mayaracvargas@hotmail.com
}

\begin{abstract}
Resumo
Introdução: Crianças prematuras tendem a apresentar atrasos no desenvolvimento neuropsicomotor devido à imaturidade e à propensão de lesões no sistema nervoso central. Objetivo: Descrever o perfil epidemiológico e motor de crianças atendidas no follow-up da Maternidade Balbina Mestrinho (MBM) em Manaus/AM, verificando a associação entre diferentes fatores socioambientais e clínicos com o desenvolvimento motor (DM). Métodos: Foram avaliadas 25 crianças acompanhadas no follow-up da MBM, por meio da Escala Motora Infantil de Alberta e aplicação de questionário estruturado contendo dados clínicos e epidemiológicos. Resultados: Foi detectado que todas as crianças eram prematuras e $44 \%$ apresentaram atipicidade no DM, relacionada principalmente a idade corrigida $(p=0,015)$ e ao grau de escolaridade materna $(p=0,019)$. Conclusão: $\mathrm{O}$ elevado índice de atipicidade no DM pode estar associado ao perfil amostral, cuja prematuridade infere em fragilidade de seus sistemas. Assim, sugere-se que maiores investigações sejam realizadas, a fim de relacionar outros fatores com o DM.
\end{abstract}

Descritores: Desenvolvimento infantil; Recém-nascido; Prematuridade.

\begin{abstract}
Introduction: Premature children tend to have neuropsychomotor development delays due to immature and propensity of lesions in the central nervous system. Objective: To describe the epidemiological and motor profile of children attended in the follow-up of Maternity Hospital Balbina Mestrinho in Manaus/AM, identifying possible motor development delays and correlating them with risk factors. Methods: 25 premature child were evaluated through the Alberta Infant Motor Scale and a structured questionnaire was used with clinical and epidemiological data from the child and parentes. Results: It was detected that $44 \%$ of the children presented atipical motor development, related to age corrected $(p=0,015)$ and the degree of maternal schooling $(p=0,019)$. Conclusion: The high index in atypical motor development can be associated whit the sample profile, whose the prematurity infers in the fragility of its systems. Thus, it is suggested that further investigations be performed in order to relate other factors to motor development.
\end{abstract}

Keywords: Infant development; Newborn; Prematurity 


\section{Introdução}

O desenvolvimento motor (DM) é considerado um processo gradual e contínuo que envolve mudanças no comportamento motor, ligado aos aspectos da postura e movimento. Ele abrange muitos aspectos do comportamento humano, podendo ser classificado em "faixas etárias" para efeito de estudo teórico ${ }^{1}$.

Os primeiros anos de vida de uma criança são marcados por complexos processos de crescimento e desenvolvimento de todo o organismo e seus sistemas, destacando o aperfeiçoamento dos movimentos autônomos e voluntários, existentes nas posturas e nas habilidades psicomotoras ${ }^{2}$. A detecção precoce das intercorrências e o adequado atendimento das necessidades das crianças nos primeiros meses de vida irão refletir diretamente no seu desenvolvimento ${ }^{3}$.

O acompanhamento neonatal ou follow-up é um programa de monitoramento de crianças até os dois anos de idade que visa, entre outros aspectos, monitorar o desenvolvimento e controle motor de crianças nascidas pré-termas, as quais poderão se beneficiar de uma intervenção precoce, mediante a identificação de atraso motor ou suspeita do mesmo ${ }^{4-6}$.

Nesse sentido, escalas de avaliação são instrumentos valiosos para mensuração específica de aspectos relacionados ao desenvolvimento de habilidades motoras, de modo que a criança possa ser avaliada sobre diferentes posturas e estímulos? ${ }^{7}$. Os testes de avaliação do desenvolvimento motor utilizam critérios de seleção variados, como a idade da criança e a área a ser avaliada (força muscular, motricidade fina, motricidade ampla, fala, ou avaliação abrangente das capacidades funcionais) e agem facilitando o planejamento de ações precoces junto aos pais, médicos e terapeutas ${ }^{8}$.

A Escala Motora Infantil de Alberta (Alberta Infant Motor Scale - AIMS) foi idealizada por duas fisioterapeutas canadenses com o desígnio de estudar o desempenho motor infantil e controle postural do nascimento até o deambular sem apoio9. A escala é formada por 58 itens (representados por posturas) e o resultado da avaliação é obtido através da somatória das posturas que foram observadas e correlacionados de acordo com a idade gestacional corrigida (IGC), utilizando a curva percentílica da $\mathrm{AIMS}^{10}$.

Diante do exposto, o objetivo geral do presente trabalho consiste em descrever o perfil epidemiológico e motor de crianças atendidas no follow-up da Maternidade Balbina Mestrinho (MBM), considerada referência em atendimentos às gestações de alto risco em Manaus/AM, assim como identificar atrasos no DM e correlacionálos com os possíveis fatores de risco.

\section{Método}

O estudo foi do tipo descritivo observacional, em que foram avaliadas, no período de 08 de janeiro a 08 de fevereiro de 2018, 25 crianças nascidas na MBM e encaminhadas ao acompanhamento no follow-up, de acordo com o método Canguru. O estudo foi aprovado pelo Comitê de Ética em Pesquisa da Universidade Federal do Amazonas-UFAM (CAAE: 79522217.8.0000.5020).

A MBM é referência em atendimento às gestações de alto risco, especializada na assistência à saúde da mulher e ao recém-nascido. É participante da Rede Cegonha do Ministério da Saúde e Hospital Amigo da Criança, possuindo 10 leitos na Unidade em Terapia Intensiva Neonatal (UTIN), 20 leitos na Unidade de Cuidado Intermediário Neonatal Convencional (UCINCO), 10 leitos na Unidade de Cuidado Intermediário Canguru (UCINCA), bem como diversos atendimentos no follow-up.

As crianças selecionadas para a pesquisa preenchiam os seguintes critérios: lactentes do $1^{\circ}$ ao $4^{\circ}$ trimestre de vida (idade corrigida), estabilidade clínica e neurológica, além de viabilidade para realização da avaliação motora através da AIMS. Nenhuma criança, encaminhada ao follow-up, foi excluída do estudo, tendo em vista que não apresentaram malformações ou irritabilidade que impossibilitasse a avaliação. 
Para tanto, foram avaliados os prontuários dos pacientes e aplicados questionários com os genitores, a fim de obter maiores informações através dos seguintes instrumentos: 1) Roteiro de Anamnese - composto por: nome da criança, data de nascimento, nome dos pais, idade cronológica (IC), idade corrigida (IGC), sexo, cor da pele e número de prontuário; 2) Questionário de Fatores Biológicos e Ambientais Relacionados ao DM - abordando: história pré, peri e pós-natal da gestação, idade gestacional (IG), peso ao nascer, APGAR ( $1^{\circ}$ e $5^{\circ}$ minuto), tipo de parto, necessidade de reanimação ou fototerapia, utilização de ventilação mecânica invasiva (VMI) e não invasiva (VMNI), presença de mal formações ou infecções e uso de medicamentos; 3 ) Questionário Socioeconômico - contendo: renda, estrutura familiar e nível de escolaridade dos pais.

A avaliação do desenvolvimento motor e identificação de possíveis atrasos do desenvolvimento nas crianças foram realizadas através da aplicação da AIMS. A escala foi aplicada em tempo real, por aproximadamente 30 a 40 minutos, e filmagens foram obtidas para a confirmação dos resultados encontrados.

A AIMS foi escolhida por ser uma escala validada e traduzida para o idioma português, que avalia o desenvolvimento motor em crianças até os 18 meses de idade corrigida e requer uma observação minuciosa e o mínimo de contato possível com a criança. Ela é formada por 58 imagens, subdividida em quatro posições antigravitacionais básicas: prono (21 itens), supino (9), sentado (12) e em pé (16). Cada postura observada é pontuada como 1 e as posturas não observadas equivalem a 0 . O resultado da avaliação é obtido através da somatória das posturas que foram observadas e sua correlação com a IGC nas curvas percentílicas da AIMS, que permitem categorizar o DM da criança como: desempenho motor normal (resultado maior que $25 \%$ na curva percentílica), desempenho motor suspeito (resultado entre $25 \%$ e $6 \%$ ) e desempenho motor anormal (resultado menor ou igual a $5 \%$ na curva). Para fins de análise estatística, as crianças diagnosticadas com suspeita de atraso no desempenho motor foram agrupadas àquelas que apresentaram atraso de DM, de modo a formarem o grupo de DM atípico, cujos resultados foram comparados com o grupo classificado com DM típico.

Desse modo, foi criado um banco de dados no programa Microsoft Office Excel 2016 para análises preliminares quantitativas e de distribuição descritivas dos dados. Em seguida, o processamento de dados foi realizado pelo programa SPSS (Statistical Package for the Social Sciences 22), em que todas as variáveis categóricas foram analisadas através do Teste Exato de Fisher e as variáveis contínuas por meio do Teste Mann-Whitney. Foram considerados significantes resultados com $p<0,05$. Algumas variáveis apresentaram um número amostral menor que 25 crianças, devido à ausência de informações em prontuários e/ou dados inespecíficos obtidos quando da aplicação dos questionários.

\section{Resultados}

Do total de 25 crianças, a maioria era do sexo feminino (56\%) e foram descritas por seus pais como pardas (72\%). Todas as crianças foram prematuras, cuja IG ao nascimento variou entre 25 e 36 semanas, com uma média de 32 semanas. $\mathrm{O}$ peso ao nascimento variou entre $730 \mathrm{~g}$ e $2376 \mathrm{~g}$. Um total de $48 \%$ das crianças, em fase neonatal, precisou de reanimação e fez uso de surfactante, assim como, 32\% necessitaram de VMI, 72\% VMNI, $76 \%$ necessitaram de oxigenoterapia e $80 \%$ fizeram uso de fototerapia. Em relação ao desempenho motor, 56\% apresentaram resultados compatíveis com o esperado para idade e $44 \%$ apresentaram DM atípico.

O APGAR médio no primeiro e quinto minuto foram 8 e 9 respectivamente.

A tabela 1 demonstra as características relacionadas às mães, complicações gestacionais e características biológicas das crianças avaliadas.

A idade mínima das genitoras foi de 18 anos e a máxima de 45 . Todas as mães realiza- 
Tabela 1: Características relacionadas às mães, complicações gestacionais e características biológicas das crianças avaliadas

\begin{tabular}{|c|c|c|c|c|c|c|c|}
\hline \multirow{3}{*}{ Variáveis } & \multicolumn{4}{|c|}{ Classificação AIMS } & \multirow{2}{*}{\multicolumn{2}{|c|}{ Total }} & \multirow{3}{*}{$P$} \\
\hline & \multicolumn{2}{|c|}{ Típico } & \multicolumn{2}{|c|}{ Atípico } & & & \\
\hline & $\mathrm{N}$ & $\%$ & $\mathrm{~N}$ & $\%$ & $\mathrm{~N}$ & $\%$ & \\
\hline \multicolumn{8}{|c|}{ Hemorragia Intracraniana* } \\
\hline Sim & 7 & 53,8 & 6 & 46,2 & 13 & 56,5 & 1,0 \\
\hline Não & 6 & 60 & 4 & 40 & 10 & 43,5 & \\
\hline \multicolumn{8}{|c|}{ Peso ao nascer* } \\
\hline$>1500 \mathrm{~g}$ & 8 & 57,1 & 6 & 46,9 & 14 & 63,6 & 0,65 \\
\hline$<1500 \mathrm{~g}$ & 6 & 75 & 2 & 25 & 8 & 36,4 & \\
\hline \multicolumn{8}{|c|}{ Icterícia Neonatal* } \\
\hline Sim & 7 & 58,3 & 5 & 41,7 & 12 & 75 & 1,0 \\
\hline Não & 3 & 75 & 1 & 25 & 4 & 25 & \\
\hline \multicolumn{8}{|c|}{ Uso de medicamentos durante a gestação* } \\
\hline Sim & 9 & 60 & 6 & 40 & 15 & 78,9 & 0,30 \\
\hline Não & 1 & 25 & 3 & 75 & 4 & 21,1 & \\
\hline \multicolumn{8}{|c|}{ Infecção Urinária } \\
\hline Sim & 7 & 58,3 & 5 & 41,7 & 12 & 48 & 1,0 \\
\hline Não & 7 & 53,8 & 6 & 46,2 & 13 & 52 & \\
\hline \multicolumn{8}{|c|}{ Tipo de parto } \\
\hline Normal & 3 & 33,3 & 6 & 66,7 & 9 & 36 & 0,11 \\
\hline Cesárea & 11 & 68,8 & 5 & 31,3 & 16 & 64 & \\
\hline \multicolumn{8}{|c|}{ Acidentes na gestação* } \\
\hline Sim & 2 & 40 & 3 & 60 & 5 & 22,7 & 0,60 \\
\hline Não & 11 & 64,7 & 6 & 35,3 & 17 & 77,3 & \\
\hline
\end{tabular}

ram o pré-natal, com o mínimo de 03 consultas. As complicações gestacionais mais frequentes foram as infecções do trato-urinário (ITU) (48\%), seguidas de acidentes, como quedas da própria altura e acidentes automobilísticos, hipertensão arterial (16\%) e hemorragia vaginal (16\%).

A tabela 2 expõe as variáveis socioeconômicas relacionadas com o perfil motor apresentado na AIMS, em que foi observado que o grau de escolaridade materna está diretamente relacionado ao DM dos bebês avaliados. Não foram encontrados dados significativos para os achados paternos, provavelmente relacionados à dificuldade no preenchimento das informações subsidiadas pelas mães.
Tabela 2: Varióveis socioeconômicas relacionadas com o perfil motor apresentado na AIMS.

\begin{tabular}{|c|c|c|c|c|c|c|c|}
\hline \multirow{3}{*}{$\begin{array}{c}\text { Variáveis } \\
\text { Socioeconômicas }\end{array}$} & \multicolumn{4}{|c|}{ Classificação AIMS } & \multirow{2}{*}{\multicolumn{2}{|c|}{ Total }} & \multirow{3}{*}{$\mathrm{p}$} \\
\hline & \multicolumn{2}{|c|}{ Típico } & \multicolumn{2}{|c|}{ Atípico } & & & \\
\hline & $\mathrm{N}$ & $\%$ & $\mathrm{~N}$ & $\%$ & $\mathrm{~N}$ & $\%$ & \\
\hline \multicolumn{8}{|c|}{ Escolaridade Materna } \\
\hline Educação básica & 6 & 40 & 9 & 60 & 15 & 71,4 & $0,019 *$ \\
\hline Educação Superior & 6 & 100 & 0 & 0 & 6 & 28,6 & \\
\hline \multicolumn{8}{|c|}{ Renda Familiar (Salário mínimo) } \\
\hline$<1,5$ & 4 & 44,4 & 5 & 55,6 & 9 & 42,9 & 0,67 \\
\hline$>1,5$ & 7 & 58,3 & 5 & 41,7 & 12 & 57,1 & \\
\hline
\end{tabular}

A tabela 3 apresenta a análise descritiva de variáveis clínicas relacionadas ao perfil motor dos lactentes, que, por meio do Teste MannWhitney, apresentou significância estatística em relação ao IGC com o DM. Podemos observar que, apesar da IC não ter atingido nível de significância, a média do grupo típico foi de 6,84 me-

Tabela 3: Anólise descritiva de varióveis clínicas relacionadas ao perfil motor dos lactentes

\begin{tabular}{lccccc}
\hline Variáveis & Média & $\begin{array}{l}\text { Desvio } \\
\text { Padrão }\end{array}$ & Mediana & II0 & $p$ \\
\hline IC (meses) & & & & & \\
Típico & 6,84 & 3,4 & 7,5 & $4,4-9,4$ & 0,085 \\
Atípico & 10,27 & 4,26 & 11,1 & $5,3-14,25$ & \\
\hline IGC (meses) & & & & \\
Típico & 4,70 & 3,15 & 5,8 & $2-7,3$ & $0,015^{*}$ \\
Atípico & 8,75 & 4,04 & 10,1 & $3,9-12,2$ & \\
\hline Idade Materna (anos) & & & & \\
Típico & 29 & 9,46 & 29 & $20,5-35$ & 0,809 \\
Atípico & 27 & 6,66 & 26 & $21-32$ & \\
\hline IG (meses) & & & & & \\
Típico & 31,15 & 3,31 & 32 & $29-34$ & 0,072 \\
Atípico & 33,17 & 3,12 & 30,5 & $31,5-35$ &
\end{tabular}

Peso ao Nascer (g)

$\begin{array}{llllll}\text { Típico } & 1559,23 & 480 & 1622 & 1125-1971 & 0,238\end{array}$

$\begin{array}{lllll}\text { Atípico } & 1922,50 \quad 445,41 & 2025 & 2025-2247\end{array}$

Valor de $p$ segundo Teste $U$ de Mann Whitney: ${ }^{*} p<0,05$ IIO: Intervalo interquartílico; IC: Idade cronológica; IGC: Idade corrigida; IG: Idade Gestacional; g: gramas.

Fonte: Autores, 2018. 
ses, enquanto que do grupo atípico foi de 10,27 meses, o que sugere um possível diagnóstico de atraso no DM melhor evidenciado em idades mais avançadas, sobretudo quando adequadamente corrigidas.

\section{Discussão}

Venturella et al. ${ }^{11}$ estudaram 90 crianças entre 0 a 18 meses e propuseram investigar a diferença entre os sexos no que se refere ao DM do nascimento até o caminhar independente. Nesse caso, observaram que a maioria dos participantes apresentaram DM adequado e concluíram que nos dois primeiros anos de vida, meninos e meninas possuem DM amplo similar, o que também corrobora com os achados do presente estudo, os quais não evidenciaram diferenças significativas para o DM entre os sexos.

Linhares et al. ${ }^{12}$ e Maia et al. ${ }^{13}$ afirmam que RNPT e com baixo peso ao nascimento possuem risco elevado para atraso do DM devido à sua maior vulnerabilidade em relação às crianças atermas. Fato que não foi evidenciado estatisticamente significativo pelo presente estudo. Contudo, observou-se uma maior proporção de atraso do DM àquelas crianças com IG entre 34 e 36 semanas.

Reis et al. ${ }^{14}$ também analisaram a relação entre o atraso motor e o baixo peso, através da AIMS, em 30 crianças de 0 a 18 meses e encontraram que o baixo peso não apresentou relação direta com o DM. Entretanto, verificaram que crianças com baixo peso moderado a grave apresentaram uma tendência ao atraso do DM, que deve ser comprovado em estudos mais aprofundados.

Em relação à IGC, observamos significância na correlação com o DM. Isto evidencia a necessidade de correção da idade para caracterização da atipicidade no desenvolvimento. Tais achados foram corroborados pelos estudos de Saccani et al. ${ }^{15}$, D’Agostino et al. ${ }^{16}$ e Fraga et al. ${ }^{17}$, os quais concluíram que o DM está intimamente relacionado com a IGC quando comparada a IC, pois através da correção da idade é possível ava- liar o RNPT de forma mais fidedigna, já que em crianças prematuras parte do desenvolvimento intrauterino não foi oportunizado.

Além disso, os achados sobre a correção da idade e sua forte relação com o DM evidenciam a necessidade da utilização da IGC como algo rotineiro, não apenas aos profissionais da saúde, mas também aos familiares e cuidadores, que tendem a comparar erroneamente o desenvolvimento da criança prematura com crianças termas, as quais não necessitam de correção.

Embora este estudo tenha apresentado índices significantes de HIC, icterícia neonatal, sepse, utilização de VMI, VNI e oxigenoterapia, não foi possível estabelecer relação causal direta desses fatores com o atraso do DM. Porém, acredita-se que tais fatores possuem impacto nesse desenvolvimento, incluindo também o APGAR no primeiro e quinto minuto, os quais apresentaram médias de 8 e 9 respectivamente, o que entra em contrassenso com os achados sobre os altos percentuais de reanimação, utilização de surfactante e necessidade de ventilação mecânica. Pressupõe, dessa forma, que os procedimentos para avaliação do APGAR possam estar apresentando vieses.

Nicolau et al. ${ }^{18}$, avaliaram 69 crianças pré-termas na alta hospitalar através do Test of Infant Motor Performance (TIMP) e concluíram que os RNPT com piores resultados de DM foram os que necessitaram de suporte ventilatório prolongado em fase neonatal. Araújo et al. ${ }^{2}$ relatam que a ventilação mecânica, oxigenoterapia e tempo de permanência em UTI estão fortemente associados aos atrasos no DM. Esses autores apontam que a explicação pode estar relacionada à produção excessiva de radicais livres após o nascimento, a qual é intensificada tanto com a oxigenoterapia quanto com a VMI, as quais podem afetar o tecido pulmonar e restringir a movimentação espontânea global, o que pode provocar alterações hemodinâmicas e favorecer lesões neurológicas.

Gomes et al. ${ }^{19}$ apontam que a icterícia neonatal é uma das alterações mais frequentes no $\mathrm{RN}$, sendo o diagnóstico e tratamento pre- 
coce da hiperbilirrubinemia neonatal um fator importante para prevenção da sua maior complicação: "Kernicterus", que é uma síndrome neurológica causada devido a impregnação de bilirrubina nas células cerebrais. No presente estudo, $44 \%$ das crianças foram diagnosticadas com icterícia neonatal, mas o dobro $(80 \%)$ fez uso de fototerapia, o que subsidia a ideia da utilização do recurso fototerapêutico como uma conduta profilática. Contudo, assim como no nosso estudo, não foram encontrados trabalhos que relacionem diretamente a fototerapia com o atraso no DM.

Em relação as características maternas, não foram encontradas mães com idades extremas, isto é, muito jovens (adolescentes) ou senis, o que pode estar relacionada com a não diferença estatística apresentada entre a idade materna e o DM dos lactentes. Sartori et al. ${ }^{20}$ realizaram um estudo com 80 bebês, de 0 a 16 meses, em uma comunidade de Caxias do Sul/RS, e encontraram equivalência dos fatores socioeconômicos, incluindo a idade materna, para com o DM.

Em outro estudo, Oliveira et al. ${ }^{1}$, avaliaram o DM e as oportunidades ambientais de lactentes de mães adolescentes. Também compararam o DM dos lactentes que moram com as avós com o desenvolvimento dos que moram apenas com as pais. Os autores deste estudo observaram que a maioria dos lactentes filhos de mães adolescentes $(58,82 \%)$ apresentou desempenho motor normal, porém abaixo da média populacional. Verificaram também que os lactentes de mães adolescentes que moram com a avó apresentaram melhor desempenho motor quando comparados com os lactentes que moram apenas com os pais, fator que pode ser justificado pelo fato de que, muitas vezes, a avó assume o papel de "mãe-substituta". Portanto, a idade materna vem sendo apontada como um possível fator de risco para o atraso DM, que pode estar relacionado a um vínculo deficiente mãe-bebê, à falta de cuidados adequados, ausência de parceiro estável, falta de emprego remunerado, baixo nível de escolaridade, início tardio ou não aderência ao pré-natal, aumento da taxa de infecção do tra- to urinário e imaturidade fisiológica do corpo, especialmente na região uterina, o que pode ocasionar a diminuição do crescimento fetal, a probabilidade do $\mathrm{RN}$ nascer com baixo peso, bem como, a fragilidade psicológica da mãe e a dificuldade socioeconômica ${ }^{21}$.

Nosso estudo também encontrou uma relação direta, estatisticamente significativa, entre o grau de escolaridade materna e o DM das crianças, o que é corroborado pelos trabalhos de Zajonz et al. ${ }^{22}$ e Fuentefria et al. ${ }^{4}$, os quais encontraram que famílias com condições socioeconômicas desfavoráveis, incluindo o baixo nível de escolaridade materna, são fatores significantes no aparecimento do DM atípico.

A maioria da literatura descreve que a prematuridade, desnutrição, deficiência de assistência médica, inexperiência materna, desestruturação e baixa renda familiar, baixa escolaridade dos pais e número elevado de irmãos são fatores de risco preditores no diagnóstico de atrasos no desenvolvimento motor, uma vez que estão relacionados à carência de estímulos adequados para o aprimoramento do desenvolvimento infantil ${ }^{8,23,24}$.

Em termos gerais, a hipótese da pesquisa, realizada junto aos bebês no follow-up da MBM, esperava identificar e correlacionar os fatores de risco ao DM, assim como traçar o perfil motor desses lactentes. Nesse sentido, foi evidenciado, através da aplicação da AIMS, uma elevada frequência $(44 \%)$ de crianças com atipicidade de DM, o que pode ser contextualizado com os achados de Zajonz et al. ${ }^{22}$, o qual avaliou 96 crianças nascidas entre 06 e 15 meses de idade na periferia de Porto Alegre/RS, identificando que $44,8 \%$ apresentaram atraso na aquisição dos marcos motores. Os autores relacionaram os achados aos fatores ambientais e sociais, pois as famílias possuíam condições socioeconômicas desfavoráveis, baixo nível de escolaridade materna, base familiar desestruturada (com ou sem presença paterna) e mães jovens, com idade materna $\leq 24$ anos, resultados similares aos encontrados na nossa amostra.Todavia, é importante salientar que nosso estudo encontrou limitações 
relativas à coleta de informações, por meio dos questionários e análises de prontuários, tendo em vista que encontramos elevada taxa de subnotificações e dificuldade na obtenção dos dados junto aos genitores.

\section{Conclusão}

Os resultados deste estudo permitiram identificar um índice elevado de atipicidade no DM em lactentes atendidos no follow-up em maternidade de referência na cidade de Manaus/ $\mathrm{AM}$, o que foi diretamente associado à idade corrigida e ao nível de escolaridade materna, além do próprio perfil amostral, o qual correspondia a uma população de risco para o desenvolvimento, não só pela prematuridade, como também devido às comorbidades apresentadas (HIC e icterícia neonatal, por exemplo). Contudo, é importante considerar que o adequado DM está associado a um conjunto de fatores, os quais variam desde aspectos clínicos até socioambientais. Assim, sugere-se que maiores investigações sejam realizadas, a fim de relacionar outros fatores com o DM, sobretudo em uma população vulnerável e visando contribuir com os avanços no assistencialismo neonatal.

\section{Referências}

1. Oliveira AS, Chiquetti EMS, Santos H. Caracterização do desenvolvimento motor de lactentes de mães adolescentes. Fisioter. Pesqui. 2013;20(4):349-54.

2. Araújo ATC, Eickmann SH, Coutinho SB. Fatores associados ao atraso do desenvolvimento motor de crianças prematuras internadas em unidade de neonatologia. Rev. Bras. Saude Mater. Infant. 2013;13(2):119-28.

3. Moreira EG, Grave MTQ. Avaliação do desenvolvimento motor de crianças prematuras nascidas em uma pequena cidade do Vale do Rio dos Sinos. Rev Destaques Acadêmicos. 2014;6(3).
4. Fuentefria RN, Silveira RC. Procianoy, R.S. Motor development of preterm infants assessed by the Alberta Infant Motor Scale: systematic review article. J Pediatr. 2017;(93)4: 328-42.

5. Lima, MCP, et al. Avaliação do desenvolvimento neuromotor de crianças nascidas a termo e prétermo nos primeiros seis meses de vida. Fisioter. Bras. 2016;14(3).

6. Nuysink J, Haastert ICV, Eijsermans MJC, KoopmanEsseboom C, Helders PJM, Vries LS et al., Prediction of gross motor development and independent walking in infants born very preterm using the Test of Infant Motor Performance and the Alberta Infant Motor Scale. Early Hum Dev. 2013;89(9):693-97.

7. Lacquaniti F, Ivanenko YP, Zago M. Development of human locomotion. Curr Opin Neurobiol. 2012;22(5):822-28.

8. Willrich A, Azevedo CCF; Fernandes JO. Desenvolvimento motor na infância: influência dos fatores de risco e programas de intervenção. Rev Neurociênc. 2009;17(1):51-6.

9. Almeida TGA, Caçola PM, Gabbard C, Correr MT, Junior GBV, Santos DCC. Comparações entre o desempenho motor e oportunidades de estimulação motora no ambiente domiciliar de lactentes residentes nas regiões Sudeste e Norte do Brasil. Fisioter. Pesqui. 2015;22(2):142-47.

10. Saccani R, Valentini NC, Pereira K. New Brazilian developmental curves and references values for the Alberta infant motor scale. Infant Behav Dev. 2016;45:38-46.

11. Venturella CB, Zanandrea G, Saccani R, Valentini NC. Desenvolvimento motor de crianças entre 0 e 18 meses de idade: diferença entre os sexos. Motricidade: FTCD/FIP-MOC. 2013;9(2):3-12.

12. Linhares MB, Carvalho AE, Machado C, Martinez FE.Desenvolvimento de bebês nascidos pré-termo no primeiro ano de vida. Paidéia. 2003;13(25):59-72.

13. Maia PC, Silva LP, Oliveira MMC, Cardoso MVLML Desenvolvimento motor de crianças prematuras e a termo- uso da Alberta Infant Motor Scale. Acta Paul Enferm. 2011;24(5):670-5.

14. Reis LA, Britto IT, Lessa RS, Freitas SP, Porto TF, Reis LA. Avaliação do desenvolvimento motor em crianças de 0 a 18 meses de idade com baixo peso. Rev Baiana de Saúde Pública. 2009;33(2):153-61.

15. Saccani R, Zanella DE, Notari VS, Valentini NC. Importância da correção da idade gestacional na avaliação motora de prematuros no primeiro ano de vida. Fisioter Bras. 2017;18(4):409-16. 
16. D’Agostino JA, Gerdes M, Hoffman C, Manning ML, Phalen A, Bernbaum J. Provider use of corrected age during health supervision visits for premature infants. J Pediatr Health Care. 2013;27(3):172-9.

17. Fraga DA, Linhares MBM, Carvalho AEV, Martinez FE. Desenvolvimento de bebês prematuros relacionado a variáveis neonatais e maternas. Psicol Estud. 2008;13(2):335-44.

18. Nicolau CM, Costa APBMC, Hazime HO, Krebs VLJ. Desempenho motor em recém-nascidos pré-termo de alto risco. Rev Bras Crescimento Desenvolvimento Humano. 2011;21(2):327-34.

19. Gomes NS, Teixeira JBA, Barichello E. Cuidados ao recém-nascido em fototerapia: o conhecimento da equipe de enfermagem. Rev Eletrônica de Enfermagem. 2010;12(2):342-7.

20. Sartori N, Saccani R, Valentini NC. Comparação do desenvolvimento motor de lactentes de mães adolescentes e adultas. Fisioter. Pesqui. 2010;17(4):306-11.
21. Ribeiro FD, Ferrari RAP, Sant'Anna FL, Dalmas JC, Girotto E. Extremos de idade materna e mortalidade infantil: análise entre 2000 e 2009. Rev Paul Pediatr. 2014;32(4): 381-8.

22. Zajonz R, Müller AB, Valentini NC. A influência de fatores ambientais no desempenho motor e social de crianças da periferia de Porto Alegre. Rev. Educ. Fís/ UEM. 2008;19(2):159-71.

23. Pereira KR, Valentini NC, Saccani R. Brazilian infant motor and cognitive development: Longitudinal influence of risk factors. Pediatrics International. 2016;58(12):1297-306.

24. Valentini NC, Saccani R. Brazilian Validation of the Alberta Infant Motor Scale. Physical therapy. 2012;92(3):440-7. 